\title{
Trajectory Tracking Control and Robustness Analysis of a Robotic Manipulator Using Advanced Control Techniques
}

\author{
Reham H. Mohammed ${ }^{\text {a }}$, Basem E. Elnaghi ${ }^{\text {b }}$, Fahmy A. Bendary ${ }^{c}$, Kamel Elserfi ${ }^{\text {d }}$ \\ ${ }^{a, b}$ Electrical Department, Faculty of Engineering, Suez Canal University, 41522, Egypt \\ ${ }^{c}$ Electrical Department, Faculty of Engineering, Banha University, 13518, Egypt \\ ${ }^{d}$ Electrical Department, Faculty of Engineering, Port said University,42526, Egypt
}

Received: 19 May 2018; Accepted: 16 October 2018; Published: 08 November 2018

\begin{abstract}
The main focuses are to design controlling systems of good disturbance, stability rejection, and small errortracking. Trajectory tracking of robot manipulators are controlled by several methodologies, but when robot manipulator works with uncertain dynamic models, some limitations of this technique appear. Concerning the control perspective, such uncertainty can be divided into two groups: the unstructured inputs (e.g. disturbance effect) and the structure dynamics (e.g. the changes of parameter). Within a small number of applications, some environments, could be unknown or unstructured, make use of robot manipulators, along with some tools of strong mechanics also can make use of new methods of control to design a controller of nonlinear robust with a reasonable performance. So in this paper we test the effect of disturbance in control the first DOF of PUMA 560 using non model based FO-Fuzzy-PID controller and compared its results with two model based controllers (CTC, ANN). Also we study the effect of change of inertias parameters in the 2 cases Model based control and non- Model based control and then discus which controller give the best results. The main objective of this paper is that the non model based FO-Fuzzy-PID is able to emulate the manipulator dynamic behaviour without the need to have a complex nonlinear mathematical model for the robot.
\end{abstract}

Index Terms: PUMA560, Quintic polynomial trajectories planning, Computed Torque Control (CTC), Artificial Neural Network (ANN), Fractional Order-Fuzzy-PID (FO-Fuzzy-PID), Fractional Order PID (FOPID).

(C) 2018 Published by MECS Publisher. Selection and/or peer review under responsibility of the Research Association of Modern Education and Computer Science.

\footnotetext{
* Corresponding author. Tel.:

E-mail address: riry4mody@yahoo.com
} 


\section{Introduction}

Different applications are making use of robot manipulators [1]. As a result of exceptionally gathering dynamics of nonlinear and time varying, the robot tracking control becomes one of the problems we face. In addition to the parameters uncertainty of both manipulators mechanical parts and the actuating systems that makes the process more complex. The design of robotic manipulators control offers a new opportunity of research for the control engineers because of the advancements achieved in the techniques based on intelligent control. Several algorithms, that are based on model controllers, are used in position control, like the method of computed torque [2], optimal control [3], Variable Structure Control (VSC) [4], Neural Networks (NNs) [5, 6], Fuzzy system [1] and a model based adaptive FOPID [7]. In general, model-based controllers need a model of ideal mechanism for the controlled manipulators, and in this way however to be very complicated and computationally time consuming, especially for higher DOF manipulators. On the other hand, in the non-model based, there is no need for the parameters data of manipulators, actuators, and subsequently there would be no need for any mathematical models as well [5].

In spite of the new advance achieved in the control area, PID is the most widely strategies of control used in industry due to the simple design and implementation [8,9]. There are four main shortcomings in the traditional PID control: noise degradation in the derivative control, error computation, over simplicity, performance loss in a linear-weighted sum form within the control law, and the resulted complications of the integral control [10]. To overhaul the performance and robustness of PID systems of control, Podlubny proposed a general sort of the PID controllers, called FOPID controllers [11]. Fractional analytics are the arithmetic field that uses non-integer order to arrange integrals and derivatives. FOPID control is a recently emerged technology that was proved better performance than PID in several applications.

Effort to merge FOPID control with fuzzy was exerted recently. The trade-off between PID parameters tuning and its terms of fractional order can be minimized by the fuzzy-logic adaptive mechanism when selecting either term adaptively.

This paper aims at tracking the trajectory control of PUMA 560 first three joints of robot manipulator using non model based FO-Fuzzy-PID controller to get a trajectory of a fine quantic polynomial and with the least state of steadiness, errors of RMS, and good disturbance rejection. An excellent joint space tracking must be granted in the controller, to a specific trajectory through providing stability and less errors of tracking [12]. Finally, the proposed controller performance FO-Fuzzy-PID is compared to the other two model based ones; Computed Torque Control (CTC) and Artificial Neural Network (ANN) respectively for the trajectory tracking task. Furthermore, to prove how effective the proposed non-model based controller is, both Root Mean Square (RMS) and Steady State Errors (SSE) are discussed.

The paper in hand is organized as follows: presentation of the robot manipulator dynamic model in the second section. The third and the fourth sections introduce trajectory tracking control of the robot arm using CTC and model based ANN respectively. The fifth section introduces the principle of FOPID, while the sixth section introduces the trajectory tracking control of robot manipulator using non-model based FO-Fuzzy-PID controller. The seventh section deals with an illustration for simulation results of all developed controllers. And the last section includes the concluding remarks.

\section{Trajectory Tracking Control Of Robot Manipulator Using Model-based PID-CTC}

Computed torque controller (CTC) is an intense nonlinear controller that broadly utilized in the robot manipulator control. It depends on linearization of the feedback, and registers the needed arm torques utilizing the control law of nonlinear feedback. The best performance of this controller is shown when all physical and dynamic parameters are known. However, in case of various dynamic parameters of the robot manipulator, there would be no adequate performance of the controller $[16,17]$. 
The application of CTC is to test its efficiency for trajectory tracking control of PUMA 560 robot manipulator. Genetic algorithm (GA) applied for tuning of PID gains $\mathrm{kp}$, $\mathrm{kd}$ and $\mathrm{K}_{\mathrm{i}}$ utilizing Integral SquareError (ISE) to guarantee ideal controlling performance at specific conditions of nominal operating, where GA

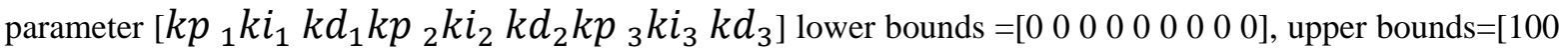
100100100100100100100 100]. The dynamic model of joint space of a robot manipulator is usually explained in the following equation (1).

$$
\tau=M(q) \tau+C(q, \dot{q})+G(q)
$$

Where $\tau$, is a $\mathrm{n} \times 1$ joint torques vector, according to the state of the joint, if it is revolute or prismatic. M (q) is a $\mathrm{n} \times \mathrm{n}$ symmetric and positive definite inertia matrix, and $\mathrm{G}(\mathrm{q})$ is a $\mathrm{n} \times 1$ gravitational torque vector, $q$ : is a $\mathrm{n} \times 1$ joint displacements vector, $\dot{q}$ : is a $\mathrm{n} \times 1$ joint velocities vector, $\ddot{q}:$ is a $\mathrm{n} \times 1$ joint accelerations vector and $n$ corresponds to the degrees number of robot freedom [14].

The linearization loop is accomplished with picking a torque $\tau$ applied to the robot as shown in "Fig. 1" [17].

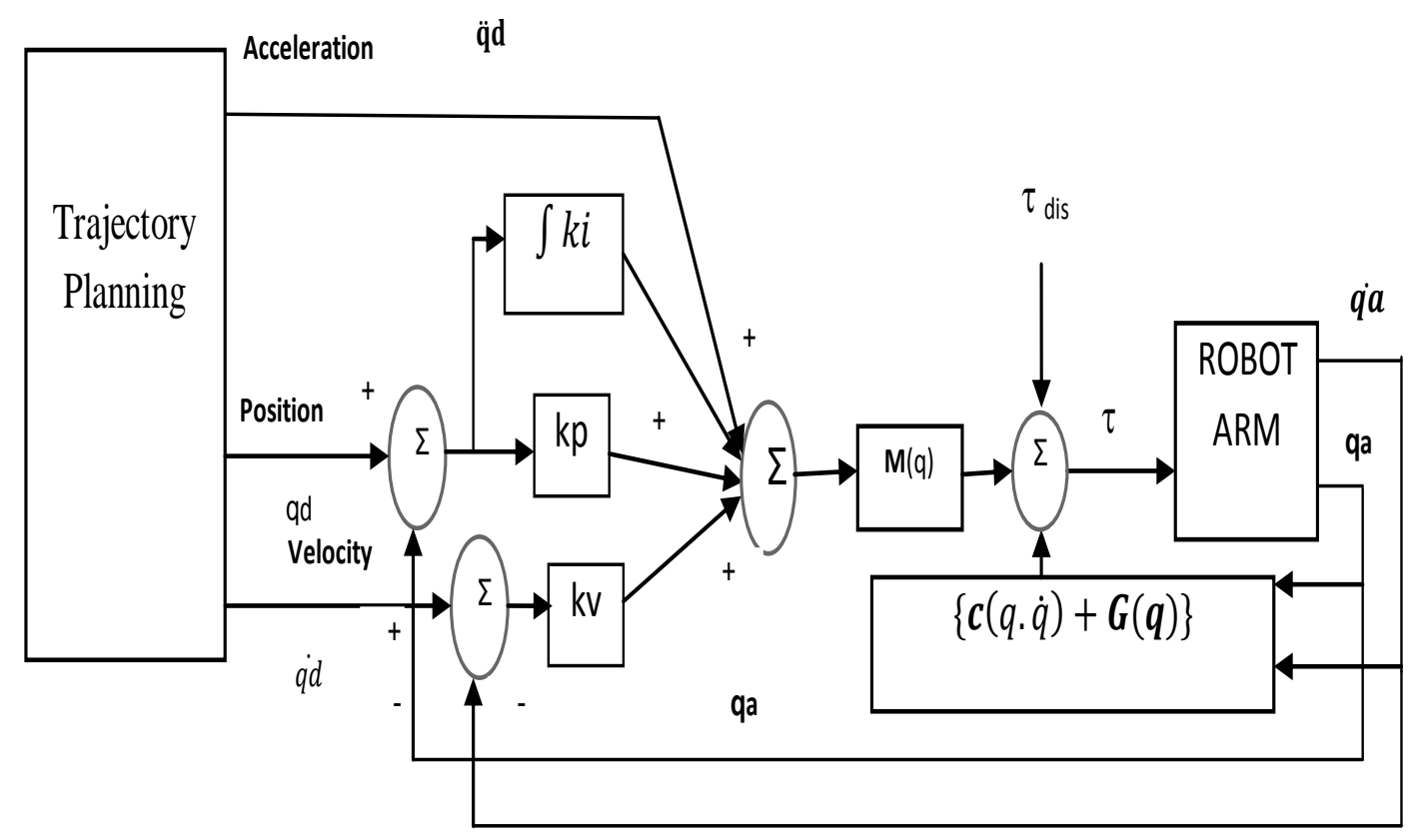

Fig.1. The Overall Block Diagram of the System based on PD-CTC Controller

$$
\tau=M(q) \tau_{0}+C(q, \dot{q})+G(q)
$$

Substituting $\tau$ in equation (1) and considering $M(q)$ that is a regular matrix, we have $n$ decoupled linear systems:

$$
\ddot{q}=\tau_{0}
$$


Where, $\tau_{0}$ is an assistant input of the choice controller. A PID control is a common decision for $\tau_{0}$, shown in the equation:

$$
\tau_{0}=\ddot{q}_{d}+k_{d}\left(\dot{q}_{d}-\dot{q}\right)+k_{p}\left(q_{d}-q\right)+k_{i} \int\left(q_{d}-q\right)
$$

Where, $k_{p}, k_{d}$ and $k_{i}$ are positive definite diagonal matrices $q_{d}, \dot{q}_{d}$ and $\ddot{q_{d}}$ : are respectively the vectors of required position, velocity, and acceleration.

By the substitution of (3) in (4), the result is the following error equation:

$$
\left[\ddot{E}+k_{d} \dot{E}+k_{p} E+k_{i} \int E(t) d t\right]=0
$$

We posture in the following:

$$
E=q d-q a \text { : Vector of the position error, }
$$

$$
\dot{E}=\dot{q}_{d}-\dot{\mathrm{q}}_{\mathrm{a}}: \text { Vector of the velocity error }
$$

The error equation (6) has answer for an error $E(t)$ that tends to zero. The aim of PID is to outline a trajectory tracking a robot arm controller by determination of a PID parameters gains $\mathrm{k}_{\mathrm{p}}, \mathrm{k}_{\mathrm{d}}$ and $\mathrm{k}_{\mathrm{i}}$ using genetic algorithm (GA).

The three gains of PID controller are calculated by ISE cost functions performance indices using GA where, the fitness value of the classical PID-GA is decreased to $1.15 \times 10^{-10}$ after 51 generations. The three PID controller gains after tuning for joint $1(\mathrm{kp} 1=45.75 \mathrm{kv} 1=61.938 \mathrm{ki1}=33.5)$ and for joint $2(\mathrm{kp} 2=51.5 \mathrm{kv} 2=56.599$ $\mathrm{ki} 2=24.5)$ and for joint $3(\mathrm{kp} 3=130.962 \mathrm{kv} 3=55.25 \mathrm{ki} 3=58.526)$ then modify this error signal to produce control input for system. Through such control input; the system is forced to produce as close as possible output to the reference level. The system is driven under control in case of the difference between reference input and instantaneous output reaches zero.

\section{Trajectory Tracking Control of Robot Arm using Model-based ANN Controller}

In this section the PID controller has been replaced with Neural Network blocks. The desired inputs position and velocity are compared with their corresponding outputs from the dynamic robot arm to determine the errors in position and velocity $\mathbf{e}_{\mathbf{p}}$ and $\mathbf{e}_{\mathbf{v}}$, respectively. Furthermore these two error signals are passed through ANN. The network consists of a three layer neural - network with two input nodes connected to ten neurons in hidden layer (with tan sigmoid transfer function) which is functioned for receiving the input data from the input layer, multiplying them according to the synaptic weights values denoted by, and forwarding the result values to the output layer (with purelin transfer function) (2-10-1).

\section{Trajectory Tracking Control of Robot Manipulator using Non-model Based FO-Fuzzy-PID Controller}

Fractional-order calculus (FOC) is a speculation of the conventional differentiation and integration that incorporate non integer orders [18]. The most well-known fractional order PID controller type is thePI $\mathrm{I}^{\mu}$. Including an order integrator $\lambda$ and an order differentiator $\mu$ where $\lambda$ and $\mu$ have the value of any real numbers. The orders of integral and derivative are not necessarily integer, but any real numbers. The FOPID controller has a transferring function explained in the following equation 8[19]: 
$G_{c}(s)=\frac{U(s)}{E(s)}=k_{p}+k_{I} \frac{1}{s^{\lambda}}+k_{D} s^{\mu},(\lambda, \mu>0)$

Where $\mathrm{G}_{\mathrm{c}}(\mathrm{s})$ is the controller transfer function, $\mathrm{E}(\mathrm{s})$ refers to an error, and $\mathrm{U}(\mathrm{s})$ refers to the controller output. The fuzzy tries to change the FOPID parameters on-line to upgrade the response of the system and also help in disturbances elimination. This method is also utilized to decrease the consumption of energy distributed in the systems of environmental control. Besides, it helps keeping a high comfortable level of occupancy. The fuzzy tries to give the controller output a nonlinear action making use of fuzzy reasoning, where the FOPID gains are tuned based on a system of fuzzy inference rather than depending on the classic methods. The FO-Fuzzy-PID controller designing process is described in detail in [20]. The robot manipulator block diagram controlled by FO-Fuzzy-PID controller is presented in Fig. 2.

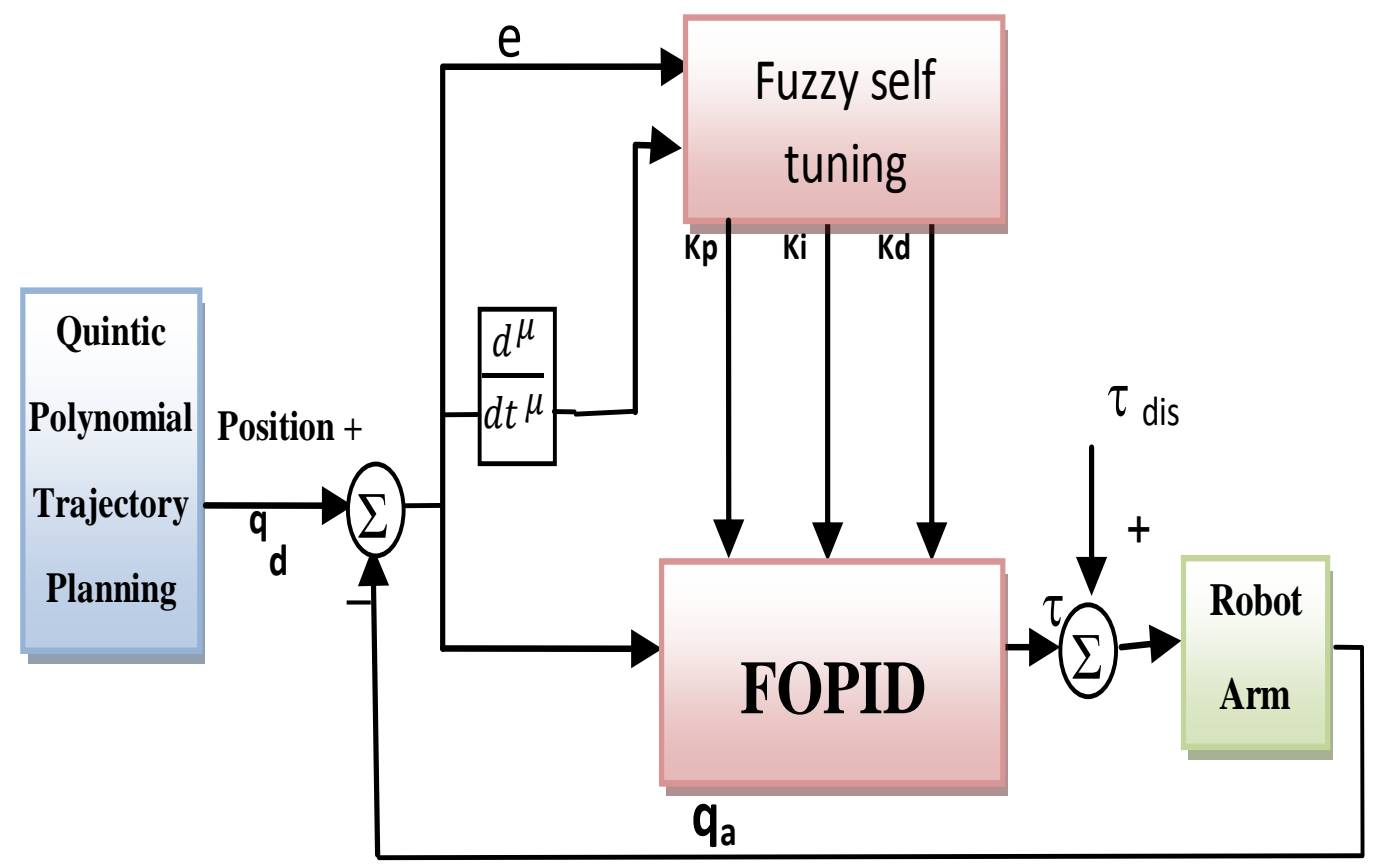

Fig.2. Structure of the block Diagram based on FO-Fuzzy-PID Controller.

\section{Simulation Results}

Through considering the PUMA 560 robot manipulator dynamics, the simulation is performed for the PUMA 560 first three DOF, by using MATLAB 2013b [14, 13]. According to the implemented studies of Armstrong and Corke; all data of the gravitational and inertial constants are presented in Appendix [4, 20] based on [13]. The main aim of implementing this simulation is to show how efficient the suggested FO-FuzzyPID is, by tracking controller compared with two model based controllers namely CTC and ANN where, all controllers tested to quintic polynomial trajectories [20]. 


\subsection{CTC Results Without Any Effect Of Disturbances Or Parameters Uncertainty}

The simulation is carried out to see its effect on the robot arm where, the position error angle for joints 1,2 and 3 of PUMA 560 robot arm controlled using CTC are shown in Figs. 3, 4 and 5 respectively.

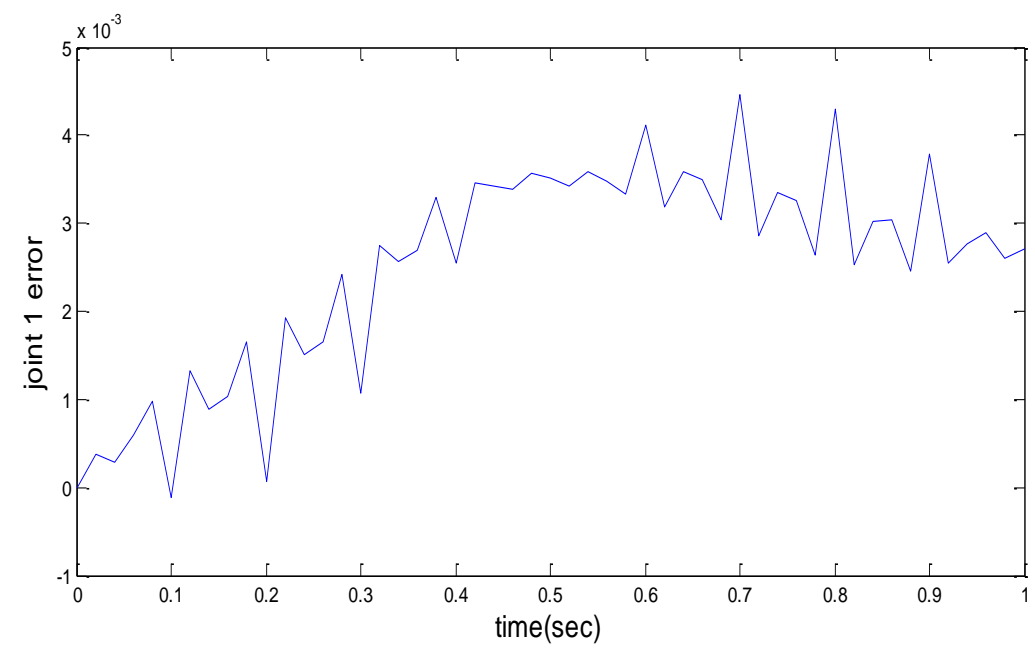

Fig.3. Error Angle for Joint 1 Controlled using PID-CTC.

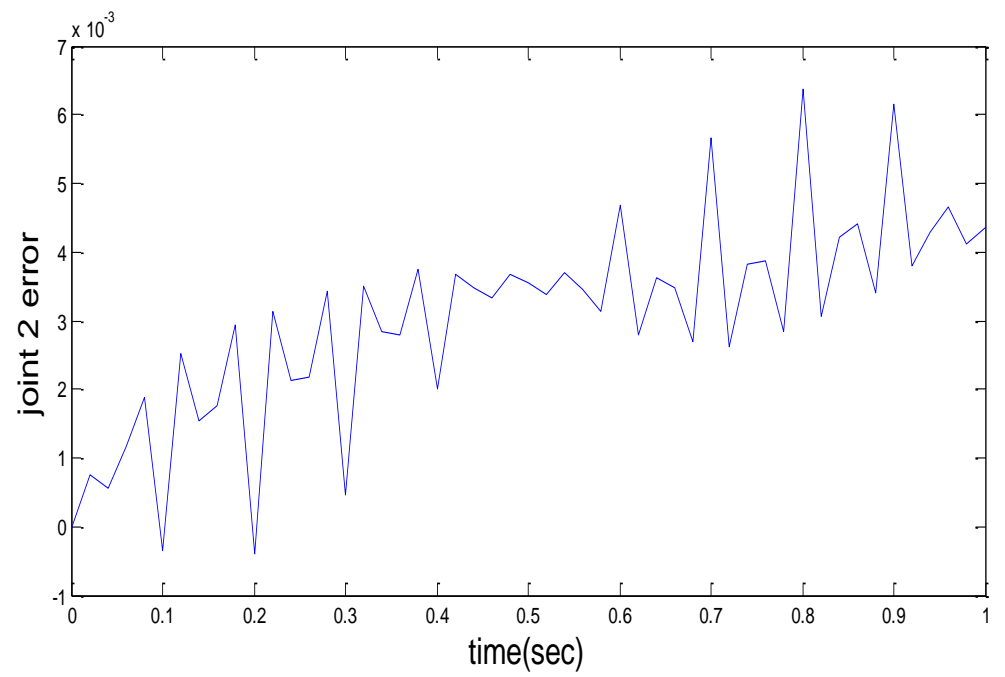

Fig.4. Error Angle for Joint 2 Controlled using PID-CTC. 


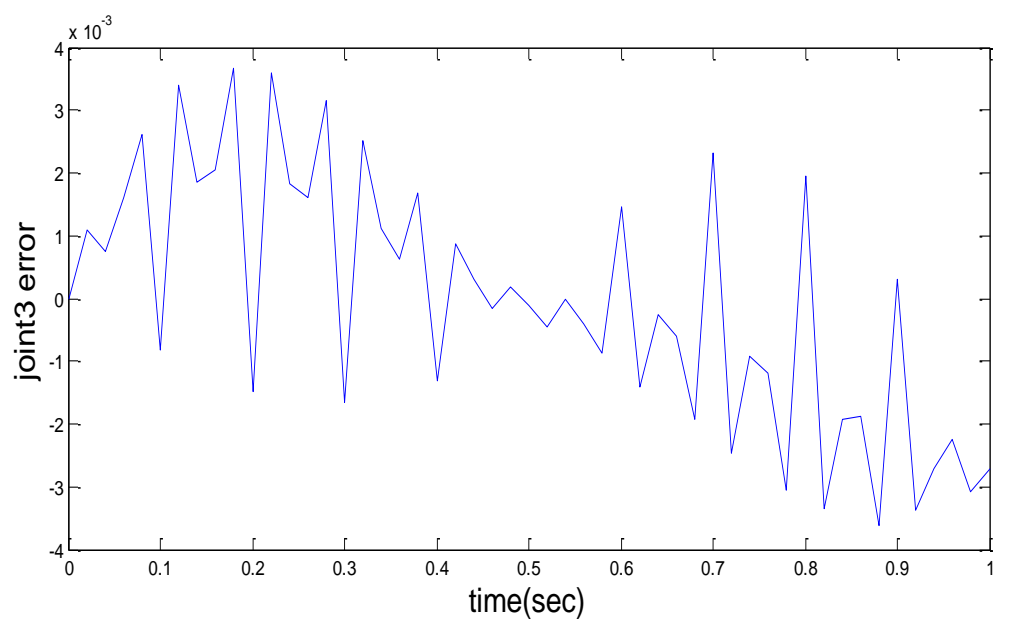

Fig.5. Error Angle for Joint 3 Controlled using PID-CTC.

Based on the results it was found with quintic polynomial trajectory tracking input that the model based CTC errors (Steady State error for joint $1=0.0002922$, joint $2=0.0003984$, joint $3=-0.001$ and $R M S$ error $=0.0002818$ ).

\subsection{ANN Results Without Any Effect of Disturbances or Parameters Uncertainty}

By simulation results it was appeared that the position error angle for joints 1, 2 and 3 of PUMA 560 robot arm controlled utilizing ANN controller are shown in Figures 6, 7 and 8 respectively.

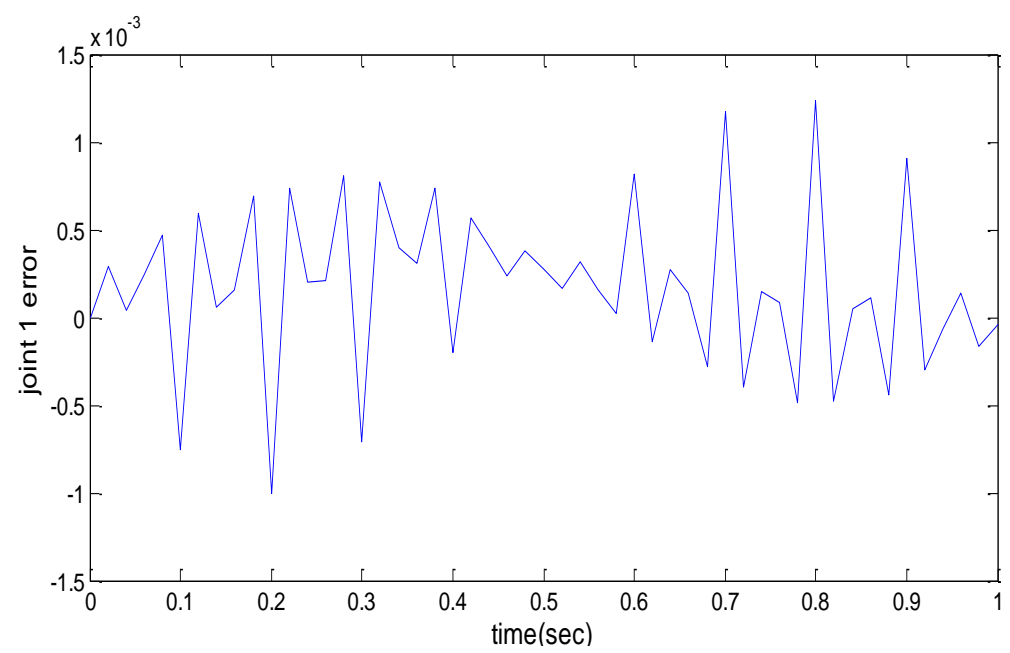

Fig.6. Error Angle for Joint 1 Controlled using model based ANN. 


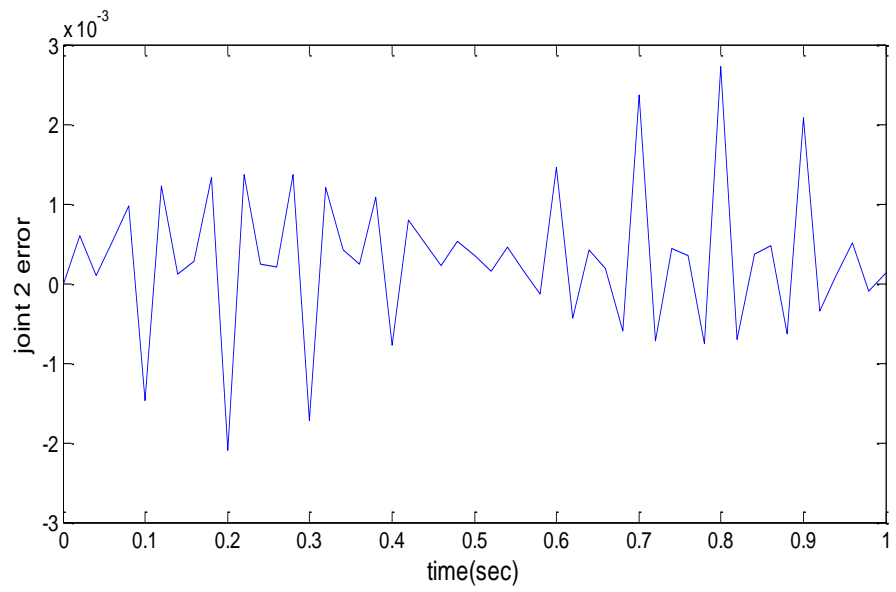

Fig.7. Error Angle for Joint 2 Controlled using model based ANN.

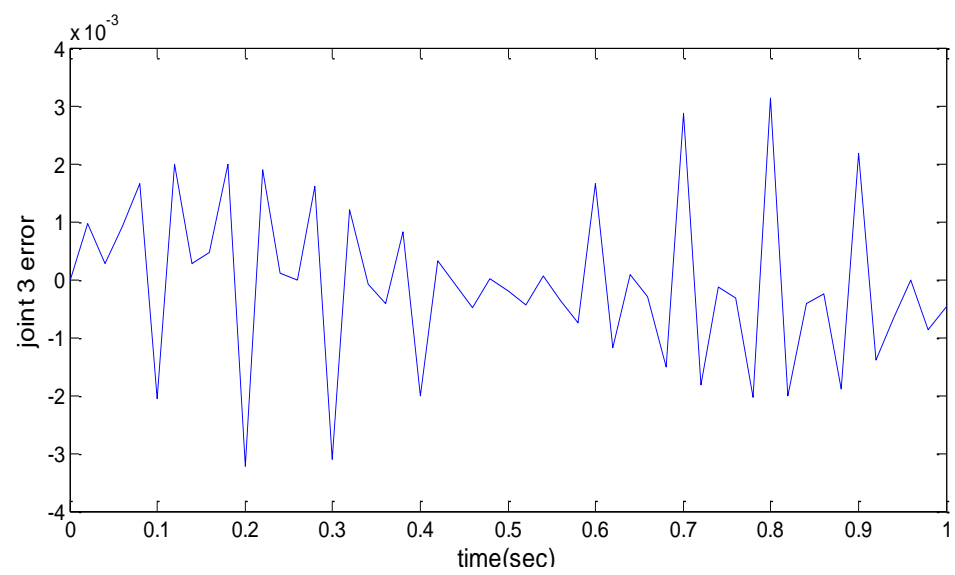

Fig.8. Error Angle between them for Joint 3 Controlled using model based ANN.

From the simulation results it was found with quintic polynomial trajectory tracking input that steady state and RMS errors in a system controlled using model based ANN's (Steady State error for joint1 $=0.0002$, joint $2=0$, joint $3=0$ and RMS error=0.0001591). Robot arm controlled using model based ANN controller has fast response and small errors compared with the CTC.

\subsection{The Non Model based FO-fuzzy-PID Controller Results Without Any Effect of Disturbances or Parameters Uncertainty}

In this section the results for joints 1, 2 and 3 of PUMA 560 robot manipulator controlled utilizing nonmodel based FO-fuzzy-PID controller with respect quintic polynomial trajectory tracking are presented .

After simulation it was noticed that the position error angle for joints 1, 2 and 3 controlled utilizing FOfuzzy-PID controllers with respect to quantic polynomial trajectory planning are appeared in Figs. 9, 10 and 11 respectively. 


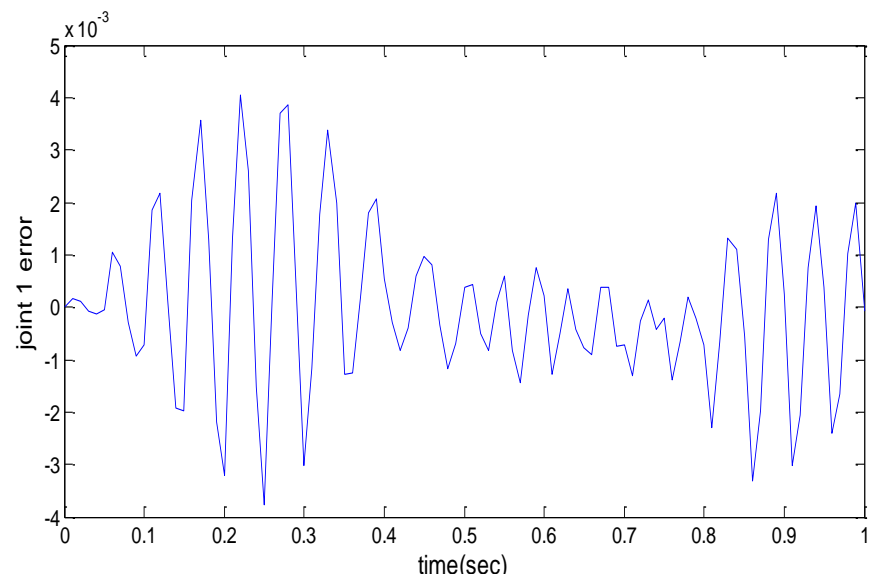

Fig.9. Error Angle for Joint 1 Controlled using Non-model based FO-Fuzzy-PID.

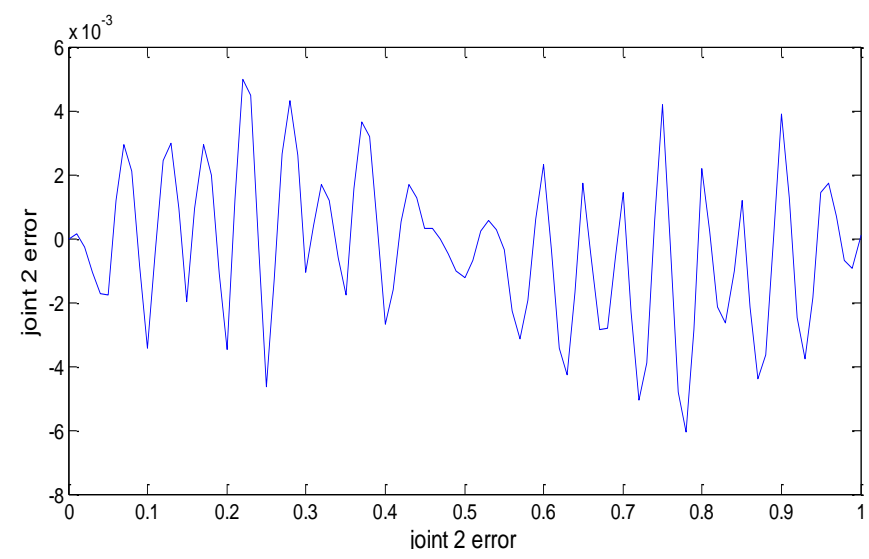

Fig.10. Error Angle for Joint 2 Controlled using Non-model based FO-Fuzzy-PID.

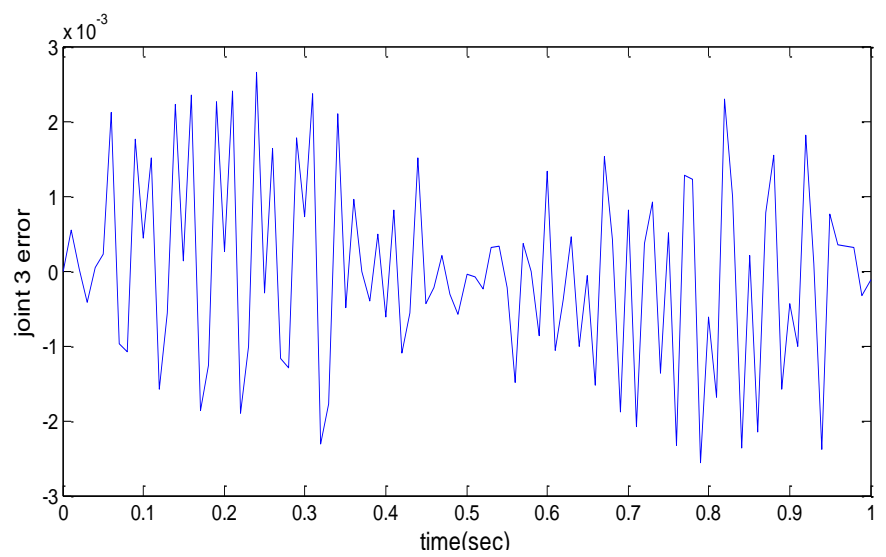

Fig.11. Error Angle for Joint 3 Controlled using Non-model based FO-Fuzzy-PID 
It was appeared with quintic polynomial tracking input that the FO-Fuzzy-PID's errors (Steady State error for joint $1=-6.953 * 10^{*} 10^{-5}$, joint $2=9.417 * 10^{-5}$, joint $3=-0.0001216$ and $\mathrm{RMS}$ error $=4.407 * 10^{-5}$ ).

\subsection{Comparisons Results between Model based Controllers (CTC, ANN) and Non-model based Controller (FO-Fuzzy-PID) with and Without Effect Of Disturbance or Parameter Uncertainty}

From the previous two cases it was observed that trajectory tracking control utilizing non model based FOFuzzy-PID give a good results compared with trajectory tracking control utilizing model based PID-CTC and ANN controller without any effect of disturbances or parameters uncertainty as shown in table 1 .

The difference between the effects of the disturbance joint torques on the joint positions in model based case can be clearly appeared in table 2 where the disturbance torque of the joints has a greater effect on the angular position of the all joints than the effect of the disturbance torque on joints angular position in case of nonmodel based. As appeared in table5 and table 3 gives a complete comparison between the two cases with effect of disturbances and model parameter uncertainty.

Due to a non-model based control methodology did not need a prerequisite knowledge of the manipulator parameters and hence no mathematical model for the manipulator was required and practically difficult to implemented. By comparing the results of the previous two cases we can conclude that robot arm control utilizing non model based FO-Fuzzy-PID controller didn't effected by the disturbance and uncertainty parameters compared with the model based control as appeared in the simulation results and tables.

Table 1. Comparison between Non Model FO- Fuzzy-PID and Model based PID-CTC, ANN, for Trajectory Tracking Control of Robot Manipulator Without Effect of Disturbances or Parameter Uncertainties.

\begin{tabular}{|l|l|l|l|l|l|}
\hline \multicolumn{2}{|l|}{ Controller type } & RMS error & SSE for j1 & SSE for j2 & SSE for j3 \\
\hline \multirow{2}{*}{ Non-model based } & $\begin{array}{l}\text { FO-Fuzzy-PID } \\
\text { controller }\end{array}$ & $4.407 * 10^{-5}$ & $-7.953^{*} 10^{-5}$ & $9.417^{*} 10^{-5}$ & -0.000121 \\
\hline \multirow{2}{*}{ Model based } & PID-CTC & 0.0002818 & 0.0002922 & 0.0003984 & -0.001 \\
\cline { 2 - 7 } & $\begin{array}{l}\text { ANN } \\
\text { controller }\end{array}$ & 0.0001591 & 0 & 0 & 0 \\
\hline
\end{tabular}

Table 2. Comparison between Non Model based FO- Fuzzy-PID and Model based PID-CTC and ANN for Trajectory Tracking Control of Robot Manipulator with Effect of Disturbances.

\begin{tabular}{|l|l|l|l|l|l|}
\hline \multicolumn{2}{|c|}{ Controller type } & \multicolumn{4}{l|}{ Disturbance value 1.0 sin $50 \mathrm{t}$} \\
\cline { 3 - 6 } & $\mathrm{J} 1$ & $\mathrm{~J} 2$ & $\mathrm{~J} 3$ & All joint \\
\hline \multirow{2}{*}{ Non-model based } & $\begin{array}{l}\text { FO-Fuzzy-PID } \\
\text { controller }\end{array}$ & 0.0001172 & 0.0001072 & $4.286^{*} 10^{-5}$ & 0.0001172 \\
\hline \multirow{2}{*}{ Model based } & PID-CTC & 0.000281 & 0.000284 & 0.0002856 & 0.0002862 \\
\cline { 2 - 6 } & ANN controller & 0.0001589 & 0.0001576 & 0.0001253 & 0.000124 \\
\hline
\end{tabular}


Table 3. Comparison between Non Model FO- Fuzzy-PID and Model based PID-CTC and ANN for Trajectory Tracking Control of Robot Manipulator with Effect of Parameter Uncertainty.

\begin{tabular}{|l|l|l|l|l|l|}
\hline \multicolumn{2}{|l|}{ Controller type } & RMSE & SSE for j1 & SSE for j2 & SSE for j3 \\
\hline Non-model based & $\begin{array}{l}\text { FO-Fuzzy-PID } \\
\text { controller }\end{array}$ & 0.0006234 & 0.001249 & 0.0006579 & 0 \\
\hline \multirow{2}{*}{ Model based } & PID-CTC & 0.002731 & -0.0004123 & -0.0007618 & 0.005554 \\
\cline { 2 - 7 } & $\begin{array}{l}\text { ANN } \\
\text { controller }\end{array}$ & 0.005054 & -0.0007479 & -0.002394 & -0.009216 \\
\hline
\end{tabular}

\section{Conclusion}

This paper investigated the possibility of improving the trajectory tracking performance of a PUMA 560 robotic manipulator utilizing non-model based FO-Fuzzy-PID controllers dependent on joint space control in its main target. Its results are compared with two model based controllers namely CTC and ANN. Also it was tested the effect of disturbance in all controllers, study the effect of change of inertias parameters in the joints of the robot arm in 2 cases Model based control and non- Model based control. The performance of each of the controllers based control strategy was compared with that of the others controllers through carrying out several simulations of the robotic arm using SIMULINK under MATLAB2013a.

From the simulation results it was concluded that:

- These results appear that non model based FO-Fuzzy-PID controller has performed a response of better, fast, and smaller errors for quintic polynomial trajectory control of robot arm than the other model based controllers without any effect of disturbance or parameters uncertainty.

- By simulation results it was observed that non model based FO-Fuzzy-PID controller performance is better than the other controllers for the external disturbance rejection.

- The fast convergence of learning enables the proposed non model based FO-Fuzzy-PID controller to adaptively adjust the parameters and keep the tracking error at a low level in spite of external disturbances and uncertain conditions.

- From the previous two cases it was observed that trajectory tracking control using non model based FOFuzzy-PID give good results compared with trajectory tracking control using model based PID-CTC and ANN controller with effect of disturbances or parameters uncertainty.

- Non model based FO-Fuzzy-PID is able to emulate the manipulator dynamic behavior without the need to have a complex nonlinear mathematical model for the robot.

\section{References}

[1] Ch. R. Kumar, K. R. Sudha, D. V. Pushpalatha and Ch. V. N. Raja , "Fuzzy C-Means Controller for a PUMA-560 Robot Manipulator", IEEE Workshop on Computational Intelligence: Theories, Applications and Future Directions, IIT Kanpur, India, July 2013, pp. 57-62.

[2] Spong, Mark W. "Vidyasagar, Robot Dynamics and Control." John and Wiley and Sons, 1989.

[3] Green, A., and Sasiadek, J.Z., "Dynamics and trajectory tracking control of a two-link robot manipulator", J. Vibr. Control10, Vol.10, 2004, PP.1415-1440.

[4] F. Piltan, S. Emamzadeh, Z. Hivand, F. Shahriyari and M. Mirzaei," PUMA-560 Robot Manipulator Position Sliding Mode Control Methods Using Matlap / Simulink and Their Integration into 
Graduate/Undergraduate Nonlinear Control, Robotics and MATLAB Courses", International Journal of Robotic and Automation, Volume (6): Issue (3), 2012, pp.167-191.

[5] Mahmoud M. Al Ashi, H. Elaydi and I. Abu Hadrous,"Trajectory Tracking Control of A 2-DOF Robot Arm Using Neural Networks", MS.c, Islamic University of Gaza, Electrical Engineering Department, Feb. 2014.

[6] K. A. EL Serafi, K. Z. Moustafa, and A. A. Sallam "An Adaptive Neuro Controller for Robotic Manipulator", IEEE Int. Conf. on Electronics, Circuits, and Systems, Cairo, December 15-18, 1997.

[7] H. Delavari, R. Ghaderi, A. Ranjbar N., S.H. HosseinNia and S. Momani, "Adaptive Fractional PID Controller for Robot Manipulator", The 4th IFAC Workshop Fractional Differentiation and its Applications. Badajoz, Spain, October 18-20, 2010, pp. 1-7.

[8] S. E. Hamamci, "An algorithm for stabilization of fractional-order time delay systems using fractionalorder PID controllers," IEEE Trans. Autom. Control, vol. 52, no. 10, Oct. 2007, pp. 1964-1969.

[9] M. Ö. Efe, "Neural network assisted computationally simple $\mathrm{PI}^{\lambda} \mathrm{D}^{\mu}$ control of a quadrotor UAV", IEEE Trans. Ind. Informat., vol. 7, no. 2, May 2011, pp. 354-361.

[10] J. Han, "From PID to active disturbance rejection control," IEEE Trans. Ind. Electron., vol. 56, no. 3, Mar. 2009, pp. 900-906.

[11] Podlubny, "Fractional-order systems and $\mathrm{PI}^{\lambda} \mathrm{D}^{\mu}$ controller," IEEE Trans. Automatic Control, vol. 44, no. 1, pp. 208-214, 1999.

[12] J. J. D'Azzo, C. H. Houpis and S. N. Sheldon, "Linear control system analysis and design with MATLAB", CRC, 2003. , Z. Alassar, H. Elaydi and I. Abuhadrous "Modeling and Control of 5DOF Robot Arm Using Supervisory Control", MS.c, Islamic University of Gaza, Electrical Engineering Department, March 2010.

[13] P.I. Corke and B. Armstrong-Helouvry, "A search for consensus among model parameters reported for the PUMA 560 robot," Proceedings of the 1994 IEEE International Conference on Robotics and Automation, Vol. 2, 1994, pp. 1608-1613.

[14] Siciliano, Bruno, and Oussama Khatib, Springer handbook of robotics: Springer Science \& Business Media, 2008.

[15] Chedmail P., Gautier M. "Optimum choice of robot actuators", Trans, of ASME, J. of Engineering for Industry, Vol. 112 , No. 4, 1990, p. 361-367.

[16] Spong, Mark W., and Mathukumalli Vidyasagar. Robot dynamics and control. John Wiley \& Sons, 2008.

[17] Bachir, Ouamri, and Ahmed-Foitih Zoubir. "Adaptive neuro-fuzzy inference system based control of PUMA 600 robot manipulator." International Journal of Electrical and Computer Engineering 2.1 (2012): 90-97.

[18] D. Xue and Y. Chen, "A Comparative Introduction of Four Fractional Order Controllers," in Proceedings of the 4th World Congress on Intelligent Control and Automation, 2002, pp. 3228-3235.

[19] Faieghi, Mohammad Reza, and Abbas Nemati. On Fractional-Order PID Design. INTECH Open Access Publisher, 2011.

[20] Reham H. Mohammed, Fahmy Bendary and Kamel Elserafi. "Trajectory Tracking Control for Robot Manipulator using Fractional Order-Fuzzy-PID Controller", International Journal of Computer Applications (0975 - 8887), Vol. 134, No.15, January 2016, pp.22-29. 


\section{Authors' Profiles}

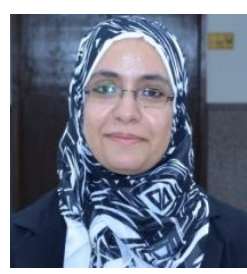

Reham H. Mohammed received her BSc in 2005 from Mansoura University, MSc in 2010 Mansoura University, and PhD in 2016 from Suez Canal University. She worked as lecturer at Department of Electrical Engineering, University of Suez Canal, Egypt. She has more than 6 scientific research papers published in prestigious international journals.

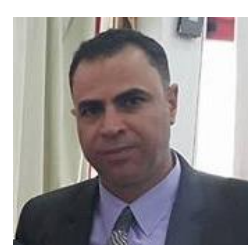

Basem Elhady received his BSc in 2003 from Port Said University, MSc in 2010 Port Said University, and PhD in 2015 from Port Said University. He worked as lecturer at Department of Electrical Engineering, University of Suez Canal, Egypt. He has more than 15 scientific research papers published in prestigious international journals.

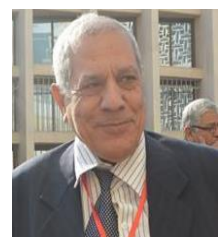

Fahmy M. Bendary worked as Prof. at Electrical Dept., Faculty of Engineering, Benha University, Shobra, Egypt. He has more than 30 scientific research papers published in prestigious international journals.

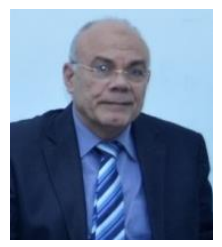

Kamel Elserafi worked as Assoc. Prof. at Electrical Dept., Faculty of Engineering, Port Said University, Egypt. He has more than 20 scientific research papers published in prestigious international journals.

How to cite this paper: Reham H. Mohammed, Basem E. Elnaghi, Fahmy A. Bendary, Kamel Elserfi,"Trajectory Tracking Control and Robustness Analysis of a Robotic Manipulator using Advanced Control Techniques", International Journal of Engineering and Manufacturing(IJEM), Vol.8, No.6, pp.42-54, 2018.DOI: 10.5815/ijem.2018.06.04 